\title{
Rupture of a suspected pancreatic lymphoepithelial cyst causing chemical peritonitis after endoscopic ultrasound guided-fine needle aspiration
}

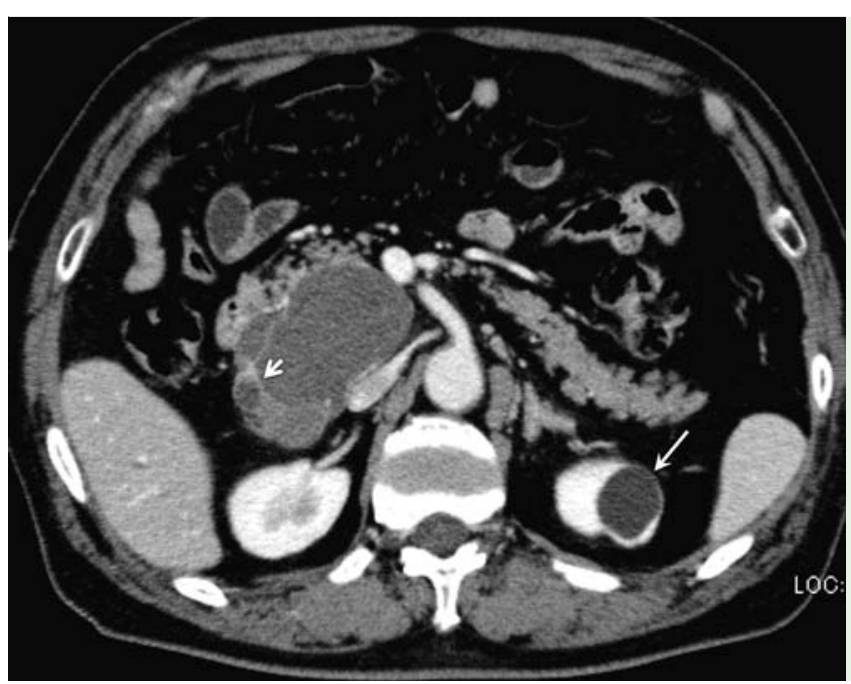

Fig. 1 Computed tomography (CT) scan from a 69-year-old man with a lesion in his pancreas showing a large low-density lesion with peripheral septa (arrow head) in the pancreatic head.

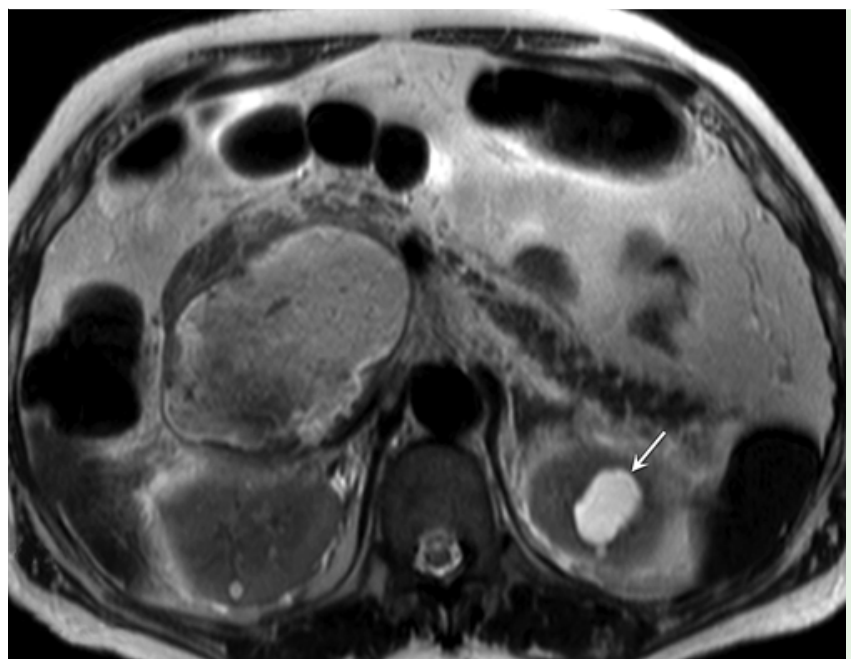

Fig. 2 T2-weighted magnetic resonance imaging (MRI) scan of the pancreatic lesion showing heterogeneity of intensity within the lesion, although the intensity is lower than that of a renal cyst (arrow).

Endoscopic ultrasound (EUS)-guided fine needle aspiration (FNA) is considered a safe technique not only for solid lesions but also for cystic lesions of the pancreas [1].

A 69-year-old man with elevated serum carbohydrate antigen (CA) 19-9 levels $(205 \mathrm{U} / \mathrm{mL})$ was referred for investigation of a lesion in the head of his pancreas. Computed tomography (CT) revealed an 8-cm, well-demarcated, low-density lesion that was compatible with a cystic lesion ( Fig.1). T2-weighted magnetic resonance imaging (MRI), however, showed heterogeneous intensities within the lesion, although the intensity was lower than that of a renal cyst ( $\bullet$ Fig. 2 ).
EUS demonstrated a pancreatic parenchyma-like echo appearance with no echolucent area ( Fig.3). Abnormal uptake of 18F-fluorodeoxyglucose (FDG) was also identified ( Fig.4), and a neoplasm derived from the pancreatic parenchyma was suspected.

EUS-FNA was performed through the duodenal bulb using a 22-gauge needle (EchoTip; Cook Medical, Winston Salem, North Carolina, USA), and the tissue obtained revealed abundant keratinized substances ( $\bullet$ Fig.5). The patient developed moderate fever 2 days after the EUS-FNA, and 2 weeks later, he felt diffuse abdominal pain. A further CT scan demonstrated a large amount of fluid in his abdominal

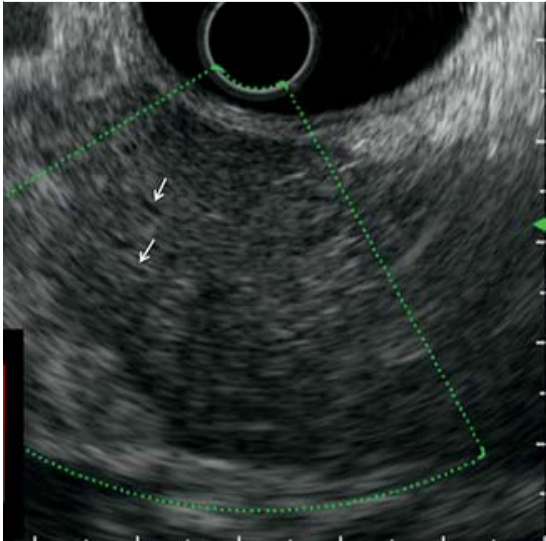

Fig. 3 Endoscopic ultrasound (EUS) image showing that the lesion demonstrated an appearance that mimicked that of pancreatic parenchyma with duct-like structures present (arrows).

cavity, and a drain was inserted ( $\bullet$ Fig. 6 ). The drained fluid was thick and yellowish-white, with extremely high levels of white blood cells ( 129750 per $\mu \mathrm{L})$, amylase (86550U/mL), and CA19-9 (4410U/mL). These findings strongly suggested rupture of a pancreatic lymphoepithelial cyst [2].

Despite administration of painkillers and antibiotics, he continued to have abdominal pain for 2 weeks, at which time the drainage stopped. Following recovery from this event, his pancreatic lesion remained unchanged in size, at $2 \mathrm{~cm}$, over the next 2 years.

Lymphoepithelial cyst is a rare pancreatic disease [2] that is sometimes seen as a heterogeneous solid mass on EUS [3]. As in the current case, diagnosis by imaging is difficult; however, the pathological and biochemical findings of the cyst aspirate are highly diagnostic $[2,3]$. Complications after EUS-FNA of pancreatic cystic lesions are infrequent $(2 \%-5 \%)[1]$, but do include serious problems such as hemosuccus pancreaticus, pancreatic ascites [4], tumor seeding [5], and, as in this case, chemical peritonitis.

Endoscopy_UCTN_Code_CPL_1AL_2AD

Competing interests: None 


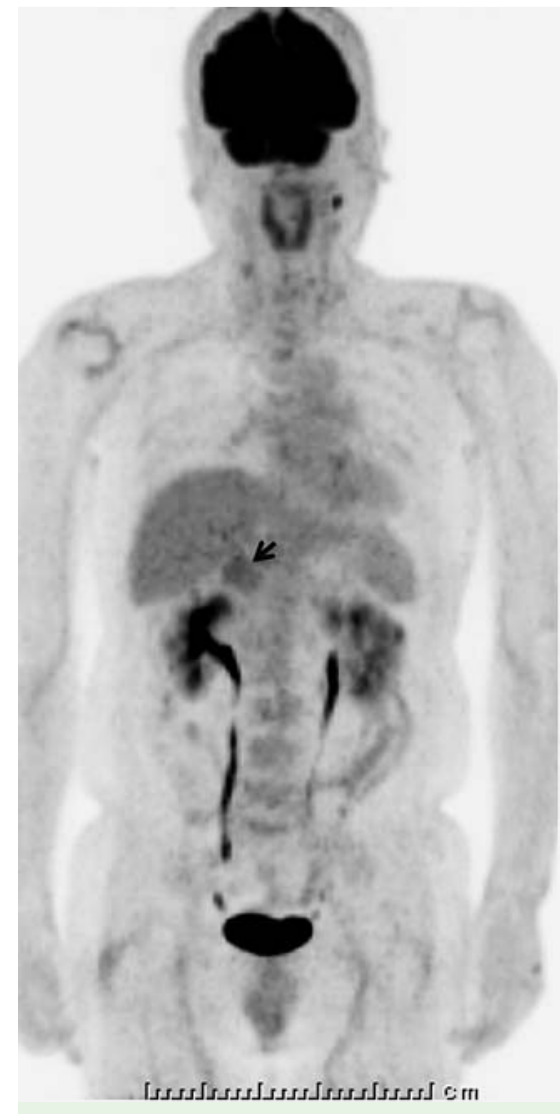

Fig. 4 Positron-emission tomography (PET) scan showing abnormal uptake of ${ }^{18} \mathrm{~F}$-fluorodeoxyglucose (FDG) at the pancreatic head $\left(S_{\text {max }}\right.$ 4.22; arrow).

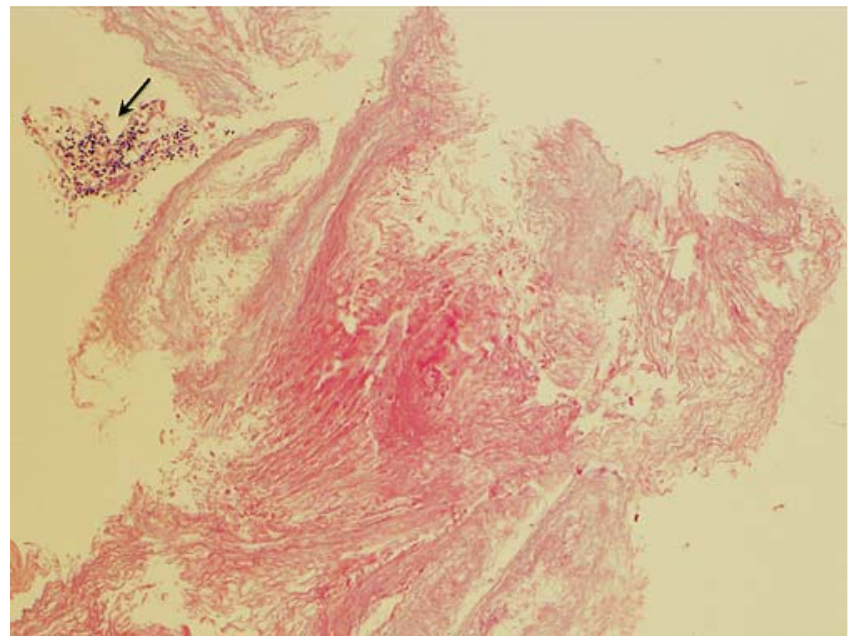

Fig. 5 Histology of the fine needle aspiration specimen showing keratinized tissues with a small fragment of epithelial tissue (arrow; hematoxylin and eosin [H\&E] stain, magnification $\times 40$ ).

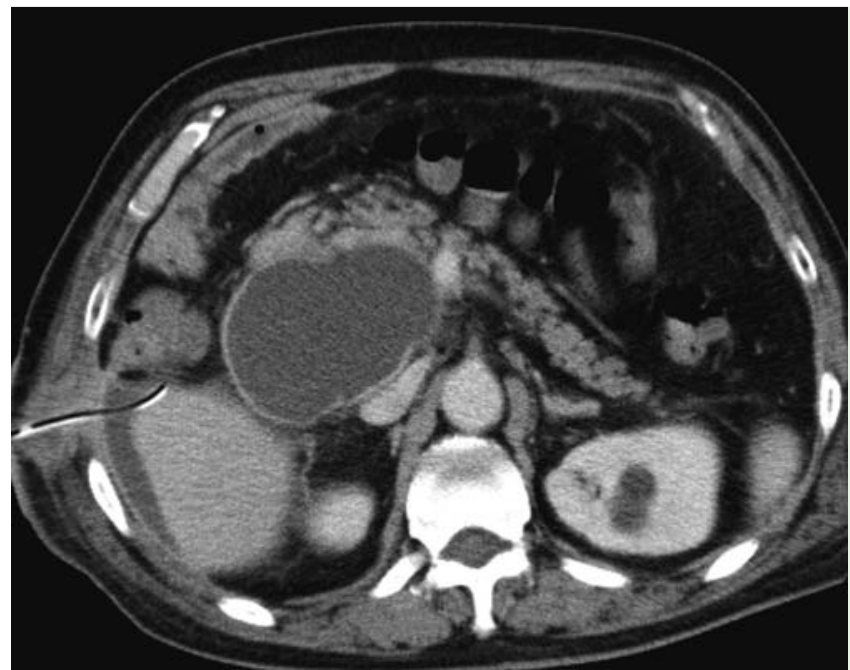

Fig. 6 Computed tomography (CT) scan performed 2 weeks later, after the patient developed abdominal pain, showing the tube that was inserted to drain the abdominal fluid that had leaked out from the ruptured pancreatic cyst causing chemical peritonitis.

\section{Hiroyuki Matsubayashi ${ }^{1}$, Shinya Sugimoto ${ }^{1}$, Yoshihiro Kishida ${ }^{1}$, Yasuyuki Tanaka', Kenichiro Imai ${ }^{1}$, Naomi Kakushima', Keiko Sasaki², Hanako Kurai ${ }^{3}$, Teichi Sugiura ${ }^{4}$, Hiroyuki Ono ${ }^{1}$}

${ }^{1}$ Department of Endoscopy, Shizuoka Cancer Center, Nagaizumi, Japan

2 Department of Pathology, Shizuoka Cancer Center, Nagaizumi, Japan

${ }^{3}$ Department of Infectious Diseases, Shizuoka Cancer Center, Nagaizumi, Japan

${ }^{4}$ Department of Hepato-pancreato-biliary Surgery, Shizuoka Cancer Center, Nagaizumi, Japan

\section{References}

1 Palazzo L, O'Toole D, Hammel P. Technique of pancreatic cyst aspiration. Gastrointest Endosc 2009; 69 (Suppl. 02): 146-S151

2 Adsay NV, Hasteh F, Cheng JD et al. Lymphoepithelial cysts of the pancreas: a report of 12 cases and a review of the literature. Mod Pathol 2002; 15: 492-501

3 Nasr J, Sanders M, Fasanella $K$ et al. Lymphoepithelial cysts of the pancreas: an EUS case series. Gastrointest Endosc 2008; 68: $170-173$

4 Babich JP, Bonasera RJ, Klein J et al. Pancreatic ascites: complication after endoscopic ultrasound-guided fine needle aspiration of a pancreatic cyst. Endoscopy 2009; 41 (Suppl. 02): E211 -E212

5 Hirooka Y, Goto H, Itoh A et al. Case of intraductal papillary mucinous tumor in which endosonography-guided fine-needle aspiration biopsy caused dissemination. J Gastroenterol Hepatol 2003; 18: $1323-$ 1324

\section{Bibliography}

Dol http://dx.doi.org/

10.1055/s-0033-1359119

Endoscopy 2014; 46: E51-E52

c) Georg Thieme Verlag KG

Stuttgart · New York

ISSN 0013-726X

\section{Corresponding author}

Hiroyuki Matsubayashi, MD

Division of Endoscopy

Shizuoka Cancer Center

1007, Nagaizumi

Suntogun

Shizuoka, 411-8777

Japan

Fax: +81-55-9895692

h.matsubayashi@scchr.jp 